\title{
Scaling of the Magnetoconductivity of Silicon MOSFETs: Evidence for a Quantum Phase Transition in Two Dimensions
}

\author{
S. A. Vitkalov, H. Zheng, K. M. Mertes, and M.P. Sarachik \\ Physics Department, City College of the City University of New York, \\ New York, New York 10031 \\ T. M. Klapwijk \\ Delft University of Technology, Department of Applied Physics, \\ 2628 CJ Delft, The Netherlands
}

(Received 30 September 2000; revised manuscript received 8 May 2001; published 3 August 2001)

\begin{abstract}
For a broad range of electron densities $n$ and temperatures $T$, the in-plane magnetoconductivity of the two-dimensional system of electrons in silicon MOSFETs can be scaled onto a universal curve with a single parameter $H_{\sigma}(n, T)$, where $H_{\sigma}$ obeys the empirical relation $H_{\sigma}=A(n)\left[\Delta(n)^{2}+T^{2}\right]^{1 / 2}$. The characteristic energy $k_{B} \Delta$ associated with the magnetic field dependence of the conductivity decreases with decreasing density, and extrapolates to 0 at a critical density $n_{0}$, signaling the approach to a zerotemperature quantum phase transition. We show that $H_{\sigma}=A T$ for densities near $n_{0}$.
\end{abstract}

DOI: $10.1103 /$ PhysRevLett.87.086401

PACS numbers: $71.30 .+\mathrm{h}, 73.40 . \mathrm{Qv}, 73.50 . \mathrm{Jt}$

Strongly interacting two-dimensional systems of electrons (or holes) have drawn intensive recent attention [1] due to their anomalous behavior: their resistance exhibits metallic temperature dependence above a critical electron [2,3] (or hole [4-6]) density $n_{c}$, raising the possibility of an unexpected metallic phase in two dimensions. An equally intriguing characteristic of these systems is their enormous response to magnetic fields applied in the plane of the electrons: the resistance increases dramatically with in-plane magnetic field and saturates to a new value above a characteristic magnetic field $H_{\text {sat }}$ on the order of several tesla [7-10]. For high electron densities, measurements of Shubnikov-de Haas oscillations [11-13] have established that the magnetic field $H_{\text {sat }}$ is the field at which full polarization of the electrons is reached. A parallel magnetic field has been shown to suppress the metallic temperature dependence [8,14]. Data obtained by Pudalov et al. [15] and by Shashkin et al. [16] indicate there is a substantial increase in the $g$ factor as the electron density is decreased toward $n_{c}$. These experimental findings all suggest that the behavior of the spins is key to understanding the enigmatic behavior of dilute, strongly interacting systems in two dimensions.

In this paper we report measurements of the temperature dependence and density dependence of the in-plane magnetoconductivity of silicon metal-oxide-semiconductor field effect transistors (MOSFETs). For a broad range of electron densities and temperatures, we show that all data for the magnetoconductance can be collapsed onto a single universal curve using a single parameter $H_{\sigma}$ which obeys an empirical relation given by $H_{\sigma}(n, T)=A(n)\left[\Delta(n)^{2}+\right.$ $\left.T^{2}\right]^{1 / 2}$. The characteristic energy $k_{B} \Delta$ associated with the response to magnetic field is found to decrease with decreasing electron density, and to exhibit critical behavior, extrapolating to 0 at a density $n_{0}$ near the critical density $n_{c}$ for the zero-field metal-insulator transition. $H_{\sigma}=A T$ for densities near $n_{0}$, so that the magnetoconductivity scales with $H / T$. Our results provide strong experimental evidence for a zero-temperature quantum phase transition at density $n_{0}$. We suggest that this is a transition to a ferromagnetically ordered state in two dimensions.

Measurements were taken on three silicon MOSFETs: the mobility $\mu \approx 30000 \mathrm{~V} / \mathrm{cm}^{2} \mathrm{~s}$ at $0.3 \mathrm{~K}$ for sample No. 1 and $\approx 20000 \mathrm{~V} / \mathrm{cm}^{2}$ s for samples No. 2 and No. 3 . Data were obtained on samples with split-gate geometry at City College to $12 \mathrm{~T}$ and at the National Magnetic Field Laboratory in fields up to $20 \mathrm{~T}$ using standard fourterminal ac techniques described elsewhere [12].

Figure 1(a) shows the longitudinal conductivity $\sigma_{x x}$ as a function of magnetic field $H_{\|}$applied parallel to the plane of a silicon MOSFET for different electron densities $n>n_{c} \approx 0.85 \times 10^{11} \mathrm{~cm}^{-2}\left(n_{c}\right.$ is the critical density for the zero-field metal-insulator transition). In agreement with earlier results, the conductivity decreases (resistivity increases) dramatically with increasing magnetic field and saturates to a value $\sigma(H=\infty)$ that is almost independent of magnetic field in fields $H>H_{\sigma}$.

Attempts [10,15-17] to obtain a collapse of the magnetoresistance onto a single scaled curve have generally yielded scaling at either low or high magnetic field, but not over the entire field range. We now report a method that allows a full mapping of the magnetoconductivity (rather than the magnetoresistivity) onto a single universal curve over the entire range of magnetic field for all data obtained at different densities $n$ and temperatures $T$, as follows. We separate the conductivity into a field-dependent contribution $[\sigma-\sigma(H=\infty)]$, which was shown [11,12] to reflect the degree of spin polarization of the electrons, and a contribution that is independent of magnetic field, $\sigma(H=\infty)$. We find that the field-dependent contribution to the conductivity, $\sigma(H=0)-\sigma(H)$, normalized to its full value, $\sigma(H=0)-\sigma(H=\infty)$ is a universal function of $H / H_{\sigma}$ 


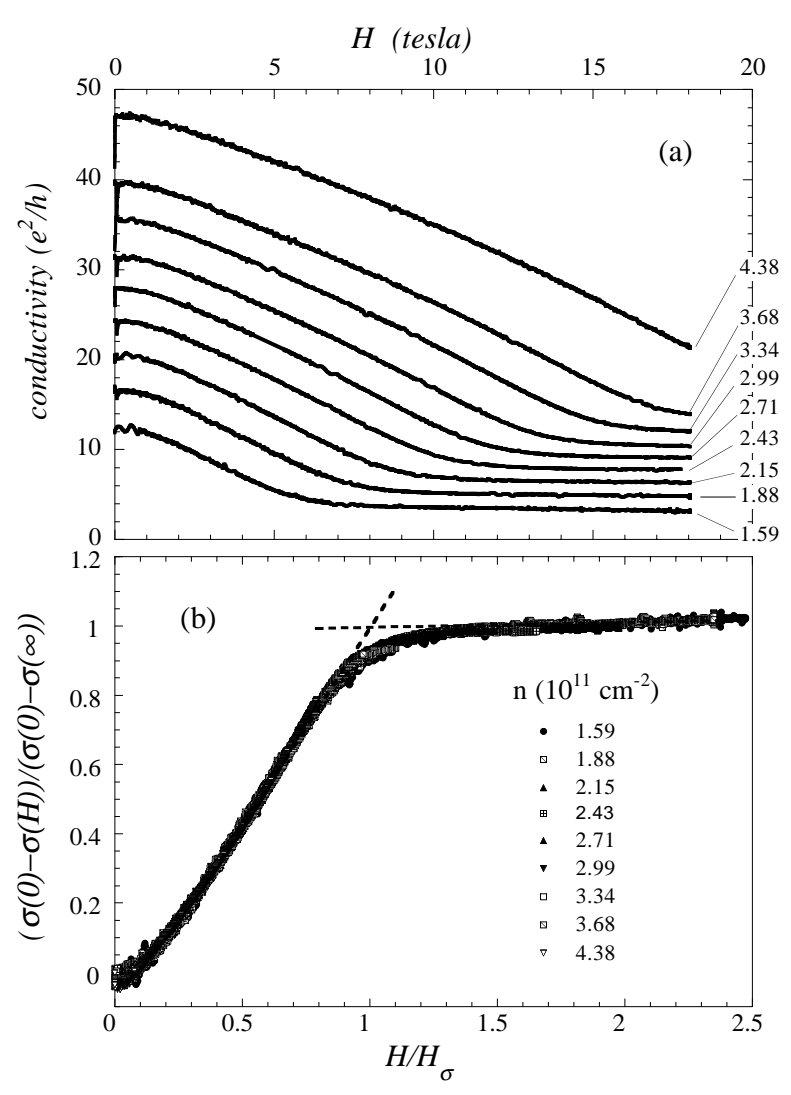

FIG. 1. (a) Conductivity versus in-plane magnetic field at different electron densities $n$ in units $10^{11} \mathrm{~cm}^{-2}$, as labeled. Data are shown for sample No. 3 at $T=100 \mathrm{mK}$. (b) Data collapse obtained by applying the scaling procedure, Eq. (1), to the curves of (a).

[18], so that

$$
\frac{\sigma(H=0)-\sigma(H)}{\sigma(H=0)-\sigma(H=\infty)}=F\left(H / H_{\sigma}\right)
$$

using a single scaling parameter $H_{\sigma}(n, T)$ which is a function of electron density $n$ and temperature $T$. Applied to the magnetoconductance curves shown in Fig. 1(a) for different electron densities, the above scaling procedure yields the data collapse shown in Fig. 1(b).

Similar scaling holds for curves obtained at different temperatures. This is demonstrated in Fig. 2, which shows the scaled magnetoconductance for sample No. 2 taken at a fixed density and different temperatures.

There are small departures from a full data collapse with temperature and as the density is varied. Possible reasons include the following: (a) the fact that the magnetoconductance is not strictly constant at high magnetic field, due possibly to orbital effects or disorder; (b) interference (weak localization) effects may become important at very high densities; (c) one expects scaling to hold only over a restricted range near a transition. Nevertheless, within an accuracy of about 5\%, the magnetoconductance curves for all electron densities and temperatures presented here can be mapped onto a universal function using a single parameter $H / H_{\sigma}$.

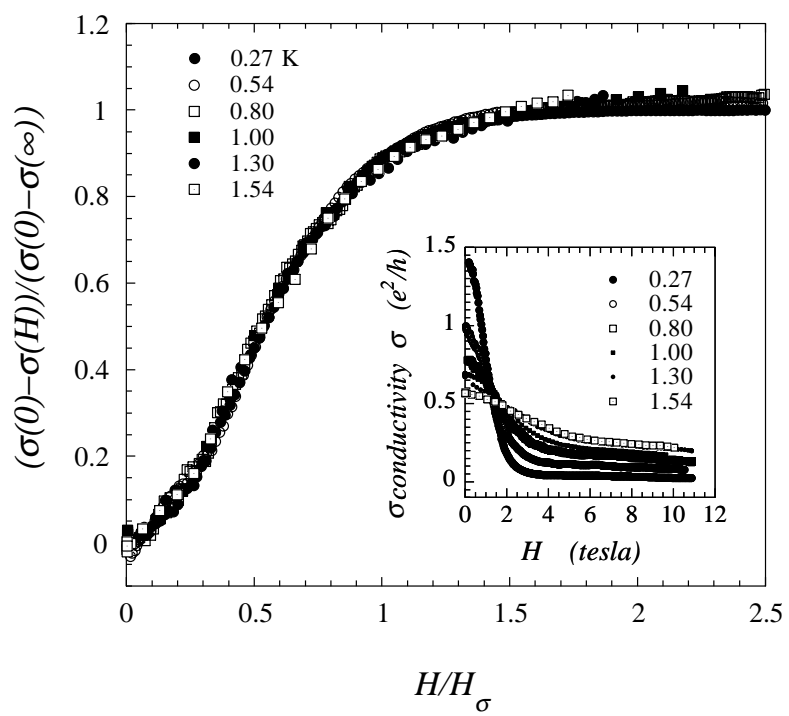

FIG. 2. Data collapse obtained by applying the scaling procedure, Eq. (1), to the in-plane magnetoconductivity of the $2 \mathrm{D}$ electrons at different temperatures for electron density $n_{s}=$ $0.94 \times 10^{11} \mathrm{~cm}^{-2}$. The inset shows the conductivity at different temperatures as a function of magnetic field. Data are shown for sample No. 2.

Having thus demonstrated that a one-parameter scaling description of the magnetoconductance holds for temperatures up to $1.6 \mathrm{~K}$ over a broad range of electron densities up to $4 n_{c}$, we report below the results of a detailed experimental investigation of the behavior of the scaling parameter, $H_{\sigma}$, as a function of temperature and electron density. In the analysis that follows, the values of $H_{\sigma}$ were determined by the intersection of the two dotted lines shown in Fig. 1(b); this intersection signals a crossover from strong field dependence at low fields, $H<H_{\sigma}$, to a conductivity that is almost independent of magnetic field at $H>H_{\sigma}$.

Figure 3(a) shows the scaling parameter $H_{\sigma}$ plotted as a function of temperature for different electron densities above the critical density for the zero-field metal-insulator transition, $n_{c}$. (Data are shown for sample No. 2, for which $n_{c}=0.85 \times 10^{11} \mathrm{~cm}^{-2}$.) The scaling parameter becomes smaller as the electron density is reduced; for a given density, $H_{\sigma}$ decreases as the temperature decreases and approaches a value that is independent of temperature, $H_{\sigma}(T=0)$. As the density is reduced toward $n_{c}$, the temperature dependence of $H_{\sigma}$ becomes stronger and its lowtemperature asymptotic value becomes smaller. Note that for electron densities below $1.36 \times 10^{11} \mathrm{~cm}^{-2}, H_{\sigma}$ is approximately linear with temperature at high $T$. The behavior of the scaling parameter $H_{\sigma}(T)$ can be approximated by an empirical fitting function:

$$
H_{\sigma}(n, T)=A(n)\left[\Delta(n)^{2}+T^{2}\right]^{1 / 2} .
$$

The solid lines in Fig. 3(a) are fits to this expression using $A(n)$ and $\Delta(n)$ as fitting parameters. As can be inferred from the slopes of the curves of Fig. 3(a), the parameter $A(n)$ is constant over most of the range and then increases measurably at lower densities (less than $20 \%$ within the 


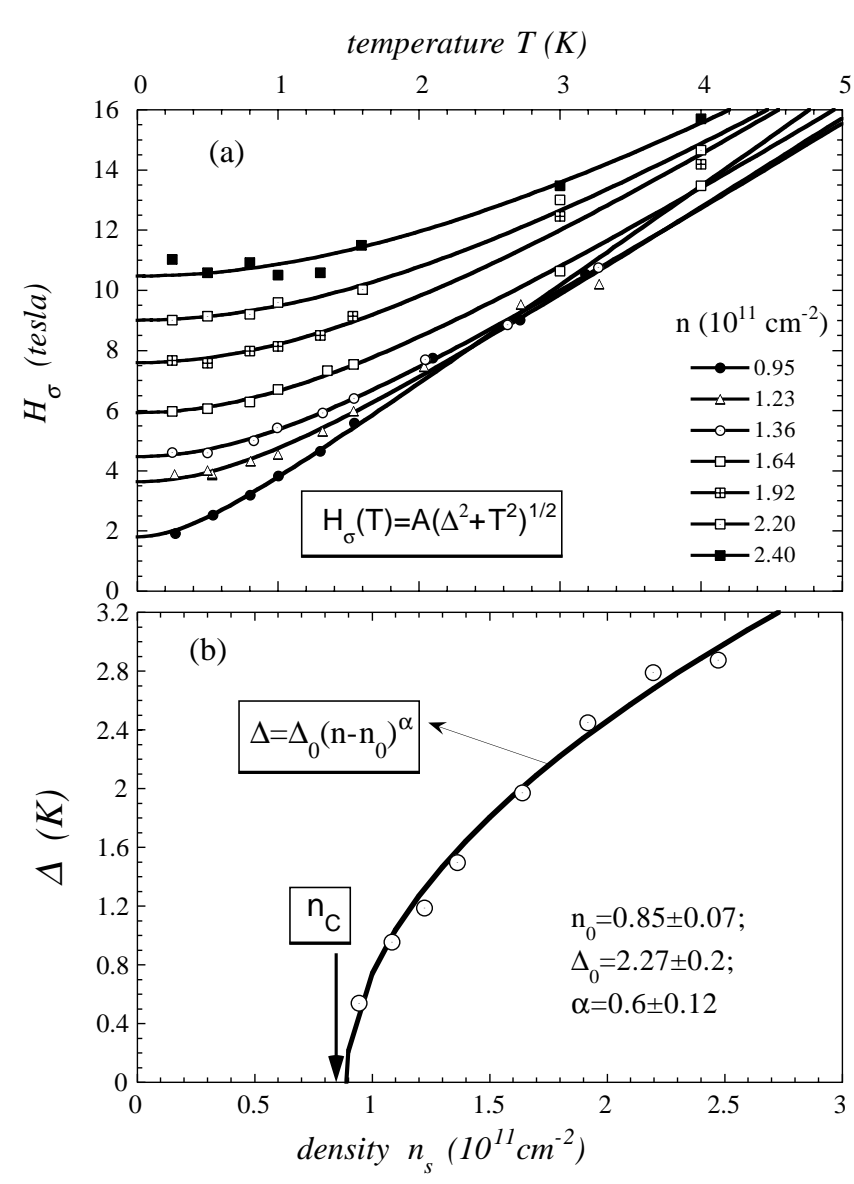

FIG. 3. (a) $H_{\sigma}$ as a function of temperature for different electron densities; the solid lines are fits to Eq. (2). (b) The parameter $\Delta$ versus electron density; the solid line is a fit to the expression $\Delta=\Delta_{0}\left(n-n_{0}\right)^{\alpha}$. Data are shown for sample No. 2 .

measured range). As shown in Fig. 3(b), the parameter $\Delta$ decreases with decreasing density and extrapolates to zero at a value $n=n_{0}$. Similar behavior was found for sample No. 1 (not shown). Within the experimental uncertainty of our measurements and of the analysis which yields $\Delta$, a fit to the critical form, $\Delta=\Delta_{0}\left(n-n_{0}\right)^{\alpha}$, yields a value for $n_{0}$ that is within $10 \%$ of the critical density $n_{c} \approx 0.85 \times$ $10^{11} \mathrm{~cm}^{-2}$ for the metal-insulator transition obtained from zero-field transport measurements. Additional measurements are required to determine whether $n_{0}$ and $n_{c}$ are the same or different densities.

The parameter $\Delta$ represents an energy $k_{B} \Delta$ and a correlation time $\tau_{H} \sim \hbar / k_{B} \Delta$. For high densities and low temperature, $T<\Delta \sim \hbar / \tau_{H}, H_{\sigma}$ is determined by $\Delta$ and the system is in the zero-temperature limit. Near $n_{0}$ the measuring temperature $T>\Delta \sim \hbar / \tau_{H}$, the field $H_{\sigma}$ is dominated by thermal effects and is not in the $T=0$ limit. At $n=n_{0}$, the energy $k_{B} \Delta$ vanishes and the correlation time $\tau_{H}$ diverges; the parameter $H_{\sigma} \rightarrow 0$ as $T \rightarrow 0$; the system exhibits critical behavior, signaling the approach to a new phase in the limit $T=0$ at a critical density $n_{0}$ [19].

As discussed earlier, a number of experiments have shown that electron spins play an important role in the response of dilute 2D systems to an in-plane magnetic field.
For high electron densities, Shubnikov-de Haas measurements [11-13] have shown directly that the unusually strong field dependence of the conductivity is associated with the polarization of the electron spins by the magnetic field. The fact that the same scaling, Eq. (1), that applies at these high densities remains valid at low electron densities implies that spin polarization continues to be a major factor in the observed magnetoconductivity as $n_{0}$ is approached. The parameter $H_{\sigma}$, the field that characterizes the system's response to in-plane magnetic field, vanishes at $n_{0}$. Thus, the behavior of the magnetoconductivity as $\Delta \rightarrow 0$ in the vicinity of $n_{0}$ indicates critical behavior for the spin susceptibility.

At $n_{0}, \Delta=0$, the scaling parameter $H_{\sigma}=A T$, and the magnetoconductance scales with $H / T$. This is illustrated explicitly in Fig. 4, where the magnetoconductivity of sample No. 1 is shown as a function of $H / T$ for a density near $n_{0}$. Magnetization that scales with $H / T$ could be associated with localized independent spins in a paramagnetic insulator, which would imply that the transition at $n_{0}$ is to a localized phase. We note, however, that at densities near $n_{0}$ the resistivity changes from weakly insulating to metallic temperature dependence, indicating that the localization length is larger than the average distance between the spins. The spins can therefore not be considered as independent.

It is possible that the critical behavior we report in this paper is due to a magnetic instability in the 2D Fermi system at density $n=n_{0}$. Support for this scenario is provided by the unusually strong temperature dependence of $H_{\sigma}$ for metallic densities near $n_{0}$ and the increase found in the value of $m^{*} g^{*}$ as the electron density is decreased $[11,15,16]$, both of which are characteristic features of a metal near a ferromagnetic instability. As shown experimentally by Okamoto et al. [11] and Vitkalov et al.

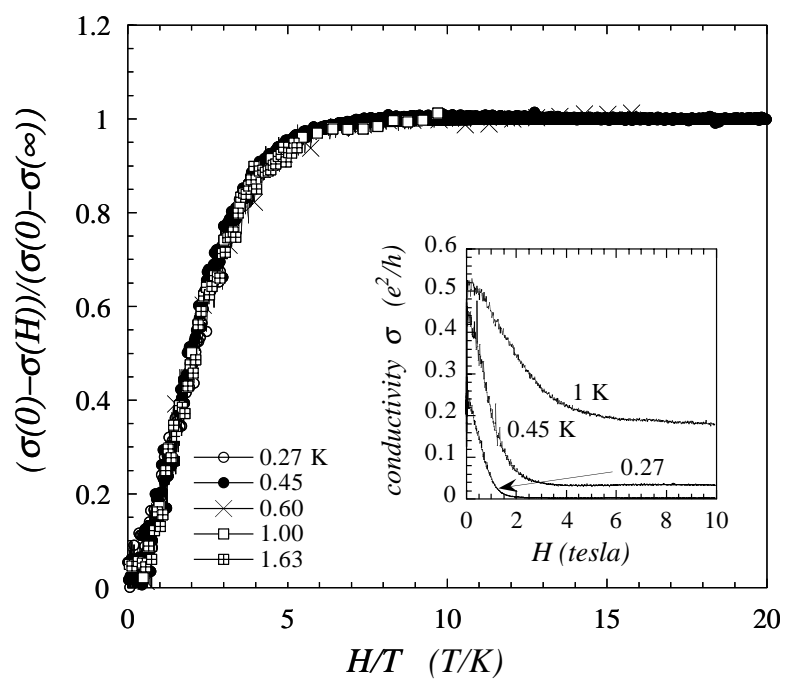

FIG. 4. The magnetoconductivity as a function of $H / T$ of sample No. 1 at a density $n=0.82 \times 10^{11} \mathrm{~cm}^{-2}$, just below the critical concentration $n_{0}$. The inset shows the conductivity as a function of magnetic field at different temperatures. 
$[12,13]$, at high electron densities the ground state of the system corresponds to complete polarization of the electron spins in high magnetic fields $H>H_{\sigma}$. The magnetoconductance continues to scale, obeying Eq. (1), as the electron density is reduced toward $n_{0}$, implying there is no change in the character of the ground state at fields $H>H_{\sigma}$ as $H_{\sigma}$ decreases. This suggests that when $H_{\sigma}$ vanishes in the limit of zero temperature at $n=n_{0}$, parallel alignment survives at least on a scale of the localization length $L_{\text {loc }}$. On this basis we suggest that a transition occurs at $n_{0}$ due to a ferromagnetic instability. This could be associated with long range order or local ordering of ferromagnetic domains or droplets.

In summary, we have shown that the in-plane magnetoconductivity of the dilute $2 \mathrm{D}$ electron system in silicon MOSFETs can be scaled with a single scaling parameter $H_{\sigma}$ over a substantial range of electron densities and temperatures. The parameter $H_{\sigma}(n, T)$ that characterizes the response of the system to a magnetic field obeys an empirical relation $H_{\sigma}=A(n)\left[\Delta(n)^{2}+T^{2}\right]^{1 / 2}$. The energy $k_{B} \Delta$ is found to be zero at a critical density $n_{0}$, indicating a quantum critical regime of the 2D dilute electron system at $n=n_{0}$. These findings provide strong evidence for a zero-temperature quantum phase transition at density $n_{0}$ near (but not necessarily the same as) the density $n_{c}$ for the apparent metal-insulator transition.

We thank B. Altshuler for suggesting the scaling procedure, S. Sachdev and D. Schmeltzer for valuable discussions, and S. V. Kravchenko and A. Shashkin for comments. S. A. V. thanks A. Larkin, B. Spivak, L. Glazman, and A. Chubukov for useful discussions. This work was supported by DOE Grant No. DOE-FG02-84ER45153. Partial support was also provided by NSF Grant No. DMR-9803440.

[1] E. Abrahams, S. V. Kravchenko, and M. P. Sarachik, Rev. Mod. Phys. 73, 251 (2001).
[2] S. V. Kravchenko, G. V. Kravchenko, J. E. Furneaux, V. M. Pudalov, and M. D'Iorio, Phys. Rev. B 50, 8039 (1994); S. V. Kravchenko, W. E. Mason, G. E. Bowker, J. E. Furneaux, V.M. Pudalov, and M. D'Iorio, Phys. Rev. B 51, 7038 (1995); S. V. Kravchenko, D. Simonian, M. P. Sarachik, Whitney Mason, and J. E. Furneaux, Phys. Rev. Lett. 77, 4938 (1996).

[3] D. Popović, A. B. Fowler, and S. Washburn, Phys. Rev. Lett. 79, 1543 (1997).

[4] P. T. Coleridge, R. L. Williams, Y. Feng, and P. Zawadzki, Phys. Rev. B 56, R12 764 (1997).

[5] Y. Hanein, U. Meirav, D. Shahar, C. C. Li, D. C. Tsui, and H. Shtrikman, Phys. Rev. Lett. 80, 1288 (1998).

[6] M. Y. Simmons, A. R. Hamilton, M. Pepper, E. H. Linfield, P. D. Rose, D. A. Ritchie, A. K. Savchenko, and T. G. Griffiths, Phys. Rev. Lett. 80, 1292 (1998).

[7] V. T. Dolgopolov, G. V. Kravchenko, A. A. Shashkin, and S. V. Kravchenko, JETP Lett. 55, 733 (1992).

[8] D. Simonian, S. V. Kravchenko, M. P. Sarachik, and V. M. Pudalov, Phys. Rev. Lett. 79, 2304 (1997).

[9] V. M. Pudalov, G. Brunthaler, A. Prinz, and G. Bauer, Pis'ma Zh. Eksp. Teor Fiz. 65, 887 (1997) [JETP Lett. 65, 932 (1997)].

[10] J. Yoon, C. C. Li, D. Shahar, D. C. Tsui, and M. Shayegan, e-print cond-mat/9907128, 1999.

[11] T. Okamoto, K. Hosoya, S. Kawaji, and A. Yagi, Phys. Rev. Lett. 82, 3875 (1999).

[12] S. A. Vitkalov, H. Zheng, K. M. Mertes, M. P. Sarachik, and T. M. Klapwijk, Phys. Rev. Lett. 85, 2164 (2000).

[13] S. A. Vitkalov, M. P. Sarachik, and T. M. Klapwijk, Phys. Rev. B 64, 073101 (2001); e-print cond-mat/0101196, 2001.

[14] K. M. Mertes, Hairong Zheng, S. A. Vitkalov, M. P. Sarachik, and T. M. Klapwijk, Phys. Rev. B 63, 041101(R) (2001).

[15] V. M. Pudalov, G. Brunthaler, A. Prinz, and G. Bauer, e-print cond-mat/0004206, 2000.

[16] A. A. Shashkin, S. V. Kravchenko, V. T. Dolgopolov, and T. M. Klapwijk, e-print cond-mat/0007402, 2000.

[17] V. T. Dolgopolov and A. Gold, JETP Lett. 71, 27 (2000).

[18] We thank B. L. Altshuler for this suggestion.

[19] S. L. Sondhi, S. M. Girvin, J. P. Carini, and D. Shahar, Rev. Mod. Phys. 69, 315 (1997). 